\title{
Performance Evaluation of Contrast Enhancement Technique for PET-CT Images in HSI Color Space
}

\author{
${ }^{1}$ Ms.J.Angelin Jeba, ${ }^{2}$ Dr.S.Nirmala Devi, \\ ${ }^{1}$ Research Scholar, ${ }^{2}$ Associate Professor, Anna University \\ Chennai,India \\ jebaangelin@gmail.com \\ nirmala_1969@hotmail.com
}

\begin{abstract}
Medical images are generally noisy due to the physical mechanisms of the acquisition process. Image

enhancement is a technique which reduces noise, removesartifacts, and preserves details in the image. Its purpose is to amplify certain image features for analysis, diagnosis, and display. In this paper, a contrast enhancement technique for the fused PET/CT (Positron Emission Tomography/ Computerized Tomography) is performed followed by the comparative analysis of filtering techniques. Some quantitative performance metrics like PSNR, SNR, CNR, MSE, RMS, AMBE and MAE are computed to evaluate the image quality.
\end{abstract}

Keywords - Contrast Enhancement, HSI space, Performance metrics.

\section{INTRODUCTION}

Medical imaging plays an important role in applications that include the clinical track of events in diagnosis, areas of planning, carrying out surgical and radiotherapy treatment

[1]. Structural imaging like Computed Tomography (CT) and Magnetic Resonance Imaging (MRI) are well suited to examine anatomical abnormalities caused by the disease. However structural imaging is not well suited for pathology detection since cellular activity is more significant. The need for functional characterization leads to the development of Positron Emission Tomography (PET) scanners, which provide the molecular activity of diseases. When combined with CT or MRI utilizing both functional and structural activity provides the higher sensitivity and specificity than is

achievable using either modality [2]. Although the sensitivity of PET scans is higher than structural images, anatomical information from another modality is still needed to localize the radiotracer uptake since the PET images are limited due to low resolution [3]. Recently PETCT combines the diagnostic information from different modalities into a single imaging device without the need for image registration. These techniques can be used to identify the disease at an earlier diagnosis with the more accurate staging of patients [4].

Fused PET-CT imaging is widely used for diagnosis, staging, treatment planning and therapy follow-up in the field of oncology. Proper contrast enhancement of fused $\mathrm{PET} / \mathrm{CT}$ images determines the segmentation and classification of the nodules as benign or malignant. Image processing plays a vital role which involves the minimum user interaction for target detection [5], pattern recognition, content-based image retrieval, medical image processing, etc. The prime task of image processing is the preprocessing stage which includes noise reduction and image enhancement. In medical image processing, the Image enhancement plays an important role to improve the visual appearance of an image or to convert the image to a form better suited for analysis by a human or machine [6]. Contrast Enhancement of an image deals with different operations of brightness increment, sharpening, blur or noise removal, etc.

The rest of the paper is organized as follows: Section 2 briefs about the study relevant to this work, Section 3 deals with the description of the proposed methodology, Section 4 discusses the experimental results and Section 5 is dealt with the conclusion.

\section{RELATED WORKS}

A number of researches have been carried out to make the system automatic in the detection of the tumor for which preprocessing of images is mandatory. From the literature it is clear that PET/CT images are affected by artifacts due to contrast variations and noise. The preprocessing stage includes both filtering and enhancement of images. K.Punithavathy et al. [7] applied preprocessing techniques such as CLAHE (Contrast Limited Adaptive Histogram Equalization) and wiener filtering in PET/CT images to reduce artifacts without affecting the image details. P.Nivetha et al. [8] applied median filtering and histogram equalization to remove the effect of poor contrast due to glare, noise and to enhance the contrast in PET/CT images. Sandeep A. Dwivedi et al. [9] used CLAHE instead of AHE (Adaptive Histogram Equalization) to improve the contrast limiting in CT images. NourhanZayed et al. [10] proposed the enhancement method of histogram equalization followed by wiener filtering to preprocess the CT images thereby to improve the quality of an image as well as make it in a form suited for further processing by human or machine.

\section{PROPOSED METHODOLOGY}

In this paper, we propose a methodology for enhancement of PET/CT images. The methodology for the proposed method is shown in figure 1.In image processing, it is often convenient to specify colors in a way that is compatible with the hardware used. The user cannot easily 
specify the desired color in the RGB model but the perceptual features such as perceived luminance (intensity), saturation and hue correlate well with human perception of color. HSI models derived from the RGB color space by coordinate transformations are better suited for human interaction. HSI family of color models use approximately cylindrical coordinates where the saturation $(\mathrm{S})$ is proportional to radial distance, the hue $(\mathrm{H})$ is a function of the angle in the polar coordinate system and the intensity (I) is the distance along the axis perpendicular to the polar coordinate plane [11].

Images that are acquired from the hospital are preprocessed to enhance the quality of fused PET/CT images. From the literature, it is clear that the quality of fused PET-CT images is affected by the artifacts due to Gaussian noise and contrast variations.Pre-processing techniques such as Wiener filtering and Contrast Limited Adaptive Histogram Equalization (CLAHE) were applied to reduce the artifacts without affecting the image details. The PET/CT image in RGB color space is converted into HSI color space [12] to specify numerically the values of hue, saturation, and intensity of a color. Initially, the RGB image of range $[0,1]$ is split into $R, G$ and $B$ vectors and pixelwise adaptive wiener filtering is applied, and is effective in removing the additive (white Gaussian) noise present in the vectors. Weiner filtering uses the neighborhood of size $\mathrm{m}$ by $\mathrm{n}$ to estimate the local mean and standard deviation. In wiener filter, the performance function is defined as:

$$
x=E\left[|e(n)|^{2}\right]
$$

This is called "mean square error criterion".

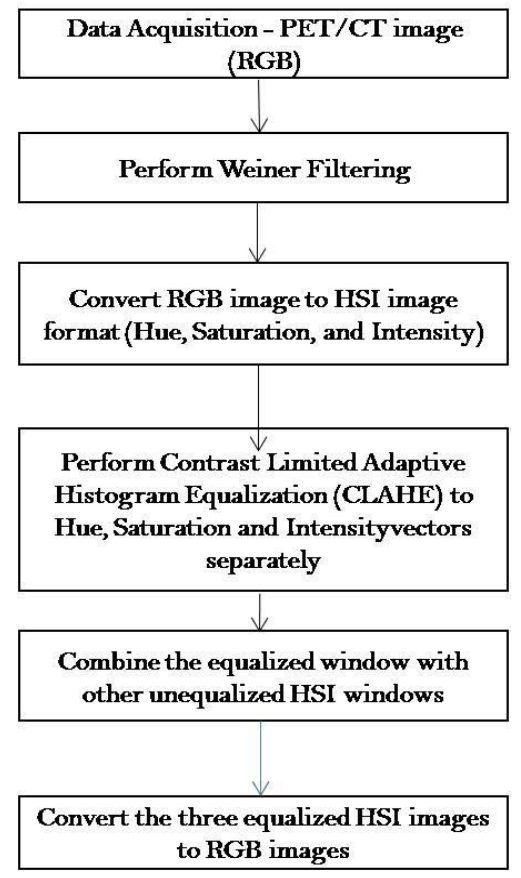

Figure 1. Overview of the proposed methodology

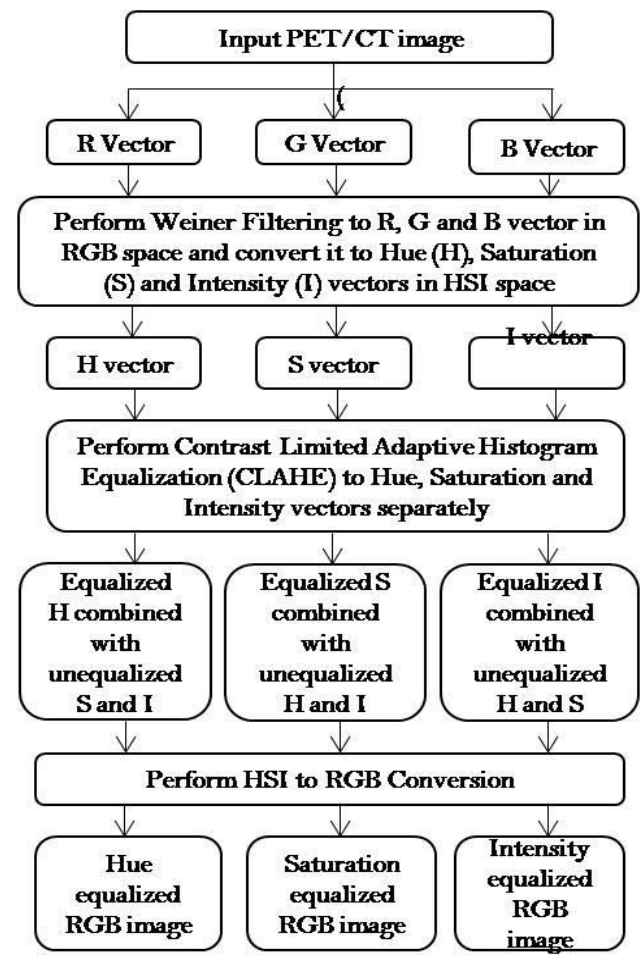

Figure 2. Contrast Enhancement Workflow

\section{A. RGB to HSI Conversion}

From the filtered R, G and B vectors the H, S and I vectors are calculated accordingly.

Hue $(H)$ :

Hue $(\mathrm{H})$ is a measure of the spectral composition of a color which is measured by the angle around the vertical axis and has a range of values between 0 and 360 degrees beginning with red at $0^{\circ}$.

$$
\begin{gathered}
\theta=\frac{\frac{1}{2}[(\mathrm{R}-\mathrm{G})+(\mathrm{R}-\mathrm{B})]}{\left\{\sqrt{\left[(\mathrm{R}-\mathrm{G})^{2}+(\mathrm{R}-\mathrm{B})(\mathrm{G}-\mathrm{B})\right]}\right\}} \\
\text { Hue }(H)=\left\{\begin{array}{l}
\theta, \quad \text { if } B \leq G \\
360-\theta, \text { if } B>G
\end{array}\right.
\end{gathered}
$$

Hue $(\mathrm{H})$ is normalized to the range $[0,1]$ by letting $\mathrm{H}=\mathrm{H} / 360^{\circ}$. Hue is not defined when the saturation $(\mathrm{S})$ is zero.

Saturation (S):

Saturation (S) refers to the relative purity or the amount of white light mixed with a hue. It is a ratio that ranges from 0 (i.e on the intensity (I) axis), extending radially outwards to a maximum value of 1 on the surface of the cylinder.

$$
\begin{array}{r}
\text { Saturation }(S)=1-\frac{3}{(R+G+B)}[\min (R, G, B)] \\
\text { Saturation }(\mathrm{S}) \text { is undefined if intensity }(\mathrm{I}) \text { is zero. }
\end{array}
$$

Intensity (I):

Intensity (I) is a measure of the relative brightness ranges between 0 and 1 . At the top and bottom of the cylinder where $\mathrm{I}=0$ and 1 respectively, Hue $(\mathrm{H})$ and 
saturation (S) component are undefined and meaningless. At any point along the Intensity (I) axis the saturation component is zero and the hue is undefined under the condition $\mathrm{R}=\mathrm{G}=\mathrm{B}$.

$$
\text { Intensity }(I)=\frac{1}{3}(R+G+B)
$$

\section{B. Contrast Enhancement}

Color image histogram equalization is performed in the HSI space to avoid undesirable shifts in image hue [13].

CLAHE is an image contrast enhancement technique for enhancing the local contrast of an image for which it operates on small regions called tiles rather than the entire image. The enhanced small regions are combined by bilinear interpolation, and the contrast in homogenous areas is limited to avoid amplification of noise [14]. The standard CLAHE method with uniform distribution is defined as:

$$
\text { Computed value, } g=\left[g_{\max }-g_{\min }\right] * p(f)+g_{7}
$$

$g_{\max }=$ Maximum pixel value, $g_{\min }=$ Minimum pixel value, $\mathrm{P}(\mathrm{f})=\mathrm{CPD}$ (Cumulative Probability Distribution)

Each tile's contrast is enhanced so that the histogram of the output region approximately matches the histogram specified by the Distribution type. The CLAHE equalization is applied to the hue, saturation and intensity channels separately. The enhanced channel is combined with the other two alternate channels to form hue enhanced HSI image, saturation enhanced HSI image and intensity enhanced HSI image. All the three image in HSI image in a range $[0,1]$ are converted back to $\operatorname{RGB}$ in $[0,1]$ range.

\section{HSI to RGB Conversion}

The hue values in $[0,1]$ range must be converted back to the un-normalized $\left(0^{\circ}, 360^{\circ}\right)$ range by letting $\mathrm{H}=360 *(\mathrm{H})$. For $R G$ sector $\left(0^{\circ}<\mathbf{H} \leq 120^{\circ}\right)$ the conversion is defined as:

$$
\begin{aligned}
& B=I *(1-S) \\
& R=I\left[1+\frac{S * \cos (H)}{\cos (60-H)}\right] \\
& G=3 I-(R+B)
\end{aligned}
$$

The conversion for the GB sector $\left(120^{\circ}<\mathbf{H} \leq \mathbf{2 4 0 ^ { \circ } )}\right.$ which implies $\mathrm{H}=\mathrm{H}-120$ is given by

$$
\begin{aligned}
& R=I *(1-S) \\
& G=I\left[1+\frac{S * \cos (H)}{\cos (60-H)}\right] \\
& B=3 I-(R+G)
\end{aligned}
$$

The conversion for the $B R$ sector $\left(240^{\circ}<\mathbf{H} \leq 360^{\circ}\right)$ which implies $\mathrm{H}=\mathrm{H}-240$ the corresponding equations are

$$
\begin{aligned}
& G=I *(1-S) \\
& B=I\left[1+\frac{S * \cos (H)}{\cos (60-H)}\right] \\
& R=3 I-(G+B)
\end{aligned}
$$

\section{Performance Metrics}

The performance of the pre-processing stage is evaluated using performance metrics such as Peak Signal to Noise Ratio (PSNR), Signal to Noise Ratio (SNR), Mean Square
Error (MSE), Root Mean Square Error (RMSE), Absolute Mean Brightness Error (AMBE), Mean Absolute Error (MAE), Contrast Difference (CD) and Contrast to Noise Ratio (CNR).

$$
\operatorname{PSNR}(d B)=10 \log _{10}\left(\frac{\text { peakval }^{2}}{M S E}\right)
$$

Peakval is the peak value.

$$
\begin{aligned}
& S N R(d B)=10 \log _{10}\left\{\frac{\sum_{y=1}^{M} \sum_{x=1}^{N}[I(x, y)]^{2}}{\sum_{y=1}^{M} \sum_{x=1}^{N}\left[I(x, y)-I^{\prime}(x, y)\right]^{2}}\right\} \\
& M S E=\left(\frac{1}{M N}\right) \sum_{y=1}^{M} \sum_{x=1}^{N}\left[I(x, y)-I^{\prime}(x, y)\right]^{2} \\
& R M S E=\operatorname{sqrt}\left[\left(\frac{1}{M N}\right) \sum_{y=1}^{M} \sum_{x=1}^{N}\left[I(x, y)-I^{\prime}(x, y)\right]^{2}\right]
\end{aligned}
$$

$I(x, y)$ and $I^{\prime}(x, y)$ are the original and filtered image respectively.

$$
A M B E=|X m-Y m|
$$

$X m$ and $Y m$ are the mean intensities of input and output images respectively.

$$
M A E=\left(\frac{1}{M N}\right) \sum_{y=1}^{M} \sum_{x=1}^{N}[n(x, y)]
$$

$n(x, y)$ gives the difference between the original and enhanced image

$$
C N R=\frac{\mu i-\mu n}{\sigma n}
$$

$\mu i$ and $\mu n$ are the mean of reference and $n(x, y)$ and $\boldsymbol{\sigma n}$ is the standard deviation of $n(x, y)$.

Contrast Difference (CD) is the objective quality measure that takes the advantage of the known characteristics of the Human Visual System (HVS). According to Michelson formula,

$$
C=\frac{f_{\max }-f_{\min }}{f_{\max }+f_{\min }}
$$

$f_{\text {max }}$ and $f_{\text {min }}$ are the maximum and minimum gray level intensities.

$$
\mathrm{CD}=|\mathrm{C} 1-\mathrm{C} 2|
$$

$C_{1}$ and $C_{2}$ are the contrast measures of original and enhanced images.

\section{RESULTS}

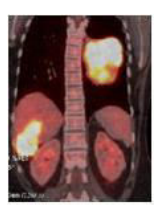

$\mathbf{a}$

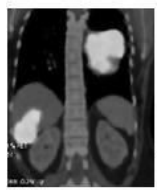

$\mathbf{f}$

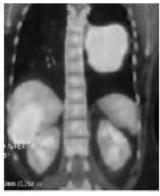

b

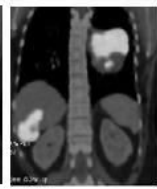

g

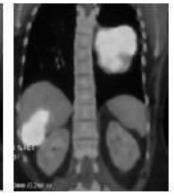

c

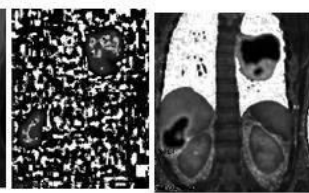

h

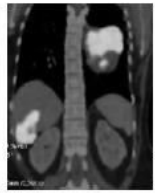

d

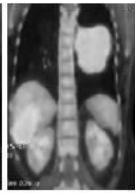

e

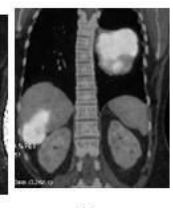

j 


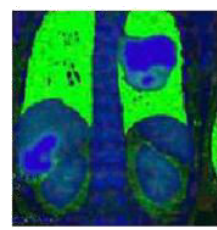

$\mathbf{k}$

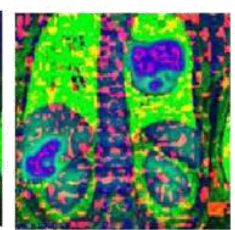

1

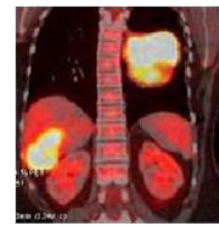

o

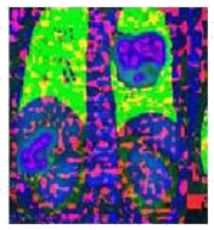

m

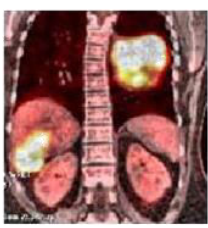

p
Figure 3. (a) PET/CT (coronal view); (b), (c) and (d) are the colour extraction of red, green and blue component; (e), (f) and (g) are the weiner filtered output of red, green and blue component; (h), (i) and (j) are the RGB to HSI Conversion of hue, saturation and Intensity component; (k), (l) and

(m) are the equalized hue, saturation and Intensity of HSI image; (n),

(o) and (p) are the HSI to RGB conversion of hue, saturation and Intensity equalized images.

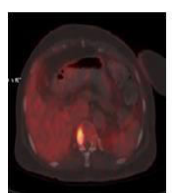

a

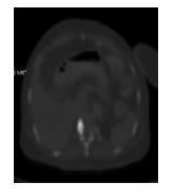

$\mathbf{f}$

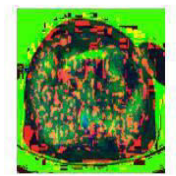

$\mathbf{k}$

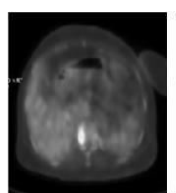

b

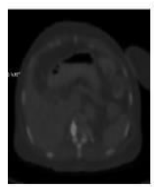

g

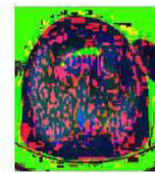

1

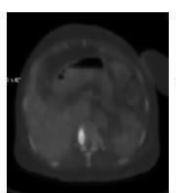

c

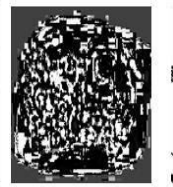

h

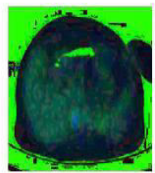

m

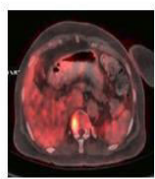

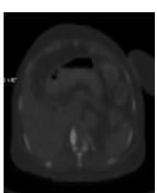

d

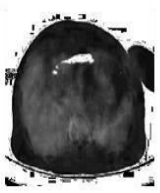

i

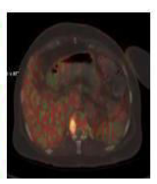

II

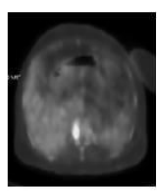

e

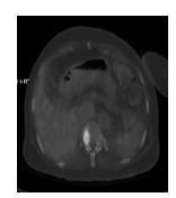

j

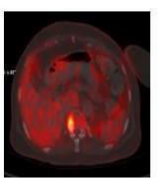

$\mathbf{0}$
Figure 4. (a) PET/CT(axial view); (b), (c) and (d) are the colour extraction of red, green and blue component; (e), (f) and (g) are the weiner filtered output of red, green and blue component; (h), (i) and (j) are the RGB to HSI Conversion of hue, saturation and Intensity component; (k), (1) and (m) are the equalized hue, saturation and Intensity of HSI image; (n), (o) and (p) are the HSI to RGB conversion of hue, saturation and Intensity equalized images.

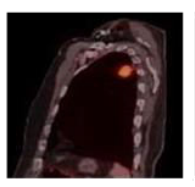

a

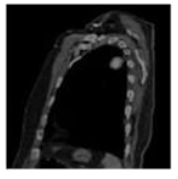

f

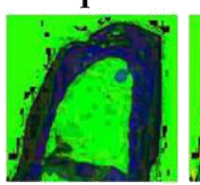

$\mathbf{k}$

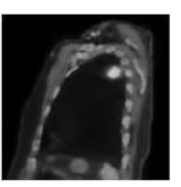

b

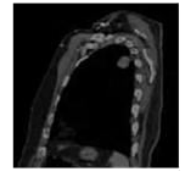

$\mathrm{g}$

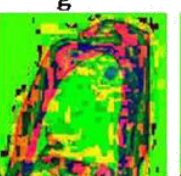

1

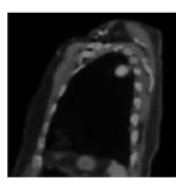

c

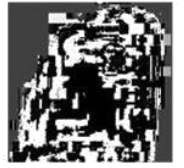

h

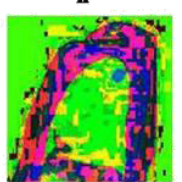

m

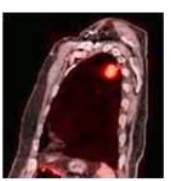

p

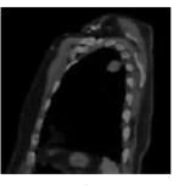

d

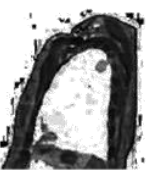

i

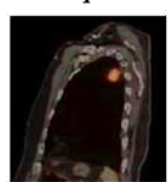

$\mathbf{n}$

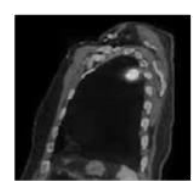

e

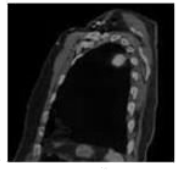

j

o

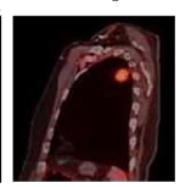

Figure 5. (a) PET/CT (Sagittal view); (b), (c) and (d) are the colour extraction of red, green and blue component; (e), (f) and (g) are the weiner filtered output of red, green and blue component; (h), (i) and (j) are the RGB to HSI Conversion of hue, saturation and Intensity component; (k), (l) and $(m)$ are the equalized hue, saturation and Intensity of HSI image; (n), (o) and (p) are the HSI to RGB conversion of hue, saturation and Intensity equalized images.

table I. Comparative Measures Of Psnr, Snr, Mse And Rmse VALUES

\begin{tabular}{|c|c|c|c|c|c|c|c|c|c|c|c|c|c|}
\hline \multirow[b]{2}{*}{ Imges } & \multirow[b]{2}{*}{ Componemets } & \multicolumn{4}{|c|}{ Medina Fiteries } & \multicolumn{4}{|c|}{ Gascinin Flteris } & \multicolumn{4}{|c|}{ Weiner Flleries } \\
\hline & & \begin{tabular}{|c|} 
PSN \\
R
\end{tabular} & $\mathbf{S N R}$ & MSE: & \begin{tabular}{|c|} 
RMS \\
$\mathbf{E}$
\end{tabular} & $\begin{array}{c}\mathbf{F N} \\
\mathbf{R}\end{array}$ & SNR & MSE & \begin{tabular}{|c|} 
RMS \\
E
\end{tabular} & $\begin{array}{c}\mathbf{P N N} \\
\mathbf{R}\end{array}$ & SNR & MSE & RMSE \\
\hline \multirow{3}{*}{$\begin{array}{c}\text { Coroual } \\
\text { vieut }\end{array}$} & Red Vector & 30.98 & 23.97 & 790E-04 & 0.0282 & 40.18 & 33.18 & $9.60 \mathrm{E} 05$ & 0.0098 & 45.43 & 38.44 & $286 \mathrm{E}-05$ & 0.0053 \\
\hline & $\begin{array}{l}\text { Greete } \\
\text { Vector }\end{array}$ & 30.82 & 20.66 & 8.29E-04 & 0.0283 & 44.28 & 35.05 & 3.73E-05 & 0.0061 & 45.79 & 35.67 & 264E-05 & 0.0051 \\
\hline & The Vector & 30.91 & 20.19 & $8.10 \mathrm{E}-04$ & 0.0285 & 40.18 & 29.48 & 9.59E-05 & 0.0098 & 45.82 & 35.12 & 262E-05 & 0.0051 \\
\hline \multirow{3}{*}{ Arialview } & Red Vector & 41.14 & 29.02 & 7.69E-05 & 0.0088 & 49.93 & 37.82 & $1.02 \mathrm{E}-05$ & 0.0032 & 5790 & 45.79 & $1.62 \mathrm{E}-06$ & 0.0013 \\
\hline & $\begin{array}{l}\text { Grees } \\
\text { Vector }\end{array}$ & 41.13 & 23.7 & 7.70E-05 & 0.0008 & 54.17 & 38.65 & $3.83 \mathrm{E} 06$ & 0.002 & 5930 & 41.97 & $1.76 \mathrm{E}-06$ & 0.0011 \\
\hline & Fine Vector & 41.15 & 23.65 & $7.68 \mathrm{E}-05$ & 0.0008 & 50.02 & 3254 & 995E 06 & 0.0032 & 59.46 & 41.99 & $1.13 \mathrm{E}-06$ & 0.0011 \\
\hline \multirow{3}{*}{$\begin{array}{l}\text { Sagittal } \\
\text { view }\end{array}$} & Red Vector & 39.01 & 27.76 & $1.26 \mathrm{E}-04$ & 0.0112 & 47.20 & 35.95 & 191E05 & 0.0044 & 49.41 & 38.16 & 1.14E-05 & 0.0034 \\
\hline & $\begin{array}{c}\text { Greet } \\
\text { Vector }\end{array}$ & 3934 & 24.12 & $1.17 \mathrm{E}-04$ & 0.0108 & 51.05 & 37.15 & 7.85E 06 & 0.0028 & 50.49 & 35.30 & 892E-06 & 0.003 \\
\hline & Vestor & 40.08 & 24.84 & $82 \mathrm{E}-05$ & 0099 & 4797 & 32.74 & $60 \mathrm{E} 05$ & 0.004 & 50.60 & 35.38 & 8.71E-06 & 0.003 \\
\hline
\end{tabular}


TABle II. Measures Of Contrast Enhancement

\begin{tabular}{|c|l|l|l|l|}
\hline Images & Measures & Hue Vector & $\begin{array}{c}\text { Saturation } \\
\text { Vector }\end{array}$ & $\begin{array}{c}\text { Intensity } \\
\text { Vector }\end{array}$ \\
\hline \multirow{3}{*}{$\begin{array}{c}\text { Coronal } \\
\text { view }\end{array}$} & $\mathrm{AMBE}$ & 0.0074 & 0.0017 & 0.1092 \\
\cline { 2 - 5 } & $\mathrm{CD}$ & 0 & 0 & 0.0043 \\
\cline { 2 - 5 } & $\mathrm{CNR}$ & 22.7915 & 34.507 & 1.6669 \\
\cline { 2 - 5 } Axial view & $\mathrm{MAE}$ & 0.0076 & 0.0046 & 0.1165 \\
\cline { 2 - 5 } & $\mathrm{CD}$ & 0 & 0.0016 & 0.0812 \\
\cline { 2 - 5 } & $\mathrm{CNR}$ & 12.3468 & 55.3283 & 0.0076 \\
\cline { 2 - 5 } & $\mathrm{MAE}$ & 0.0052 & 0.0017 & 0.0818 \\
\hline \multirow{3}{*}{$\begin{array}{c}\text { Sagittal } \\
\text { view }\end{array}$} & $\mathrm{AMBE}$ & 0.0048 & 0.0015 & 0.1016 \\
\cline { 2 - 5 } & $\mathrm{CD}$ & 0 & 0 & 0.0078 \\
\cline { 2 - 5 } & $\mathrm{CNR}$ & 13.995 & 53.297 & 0.3394 \\
\cline { 2 - 5 } & $\mathrm{MAE}$ & 0.0048 & 0.0016 & 0.1021 \\
\hline
\end{tabular}

\section{SNR PLOT}

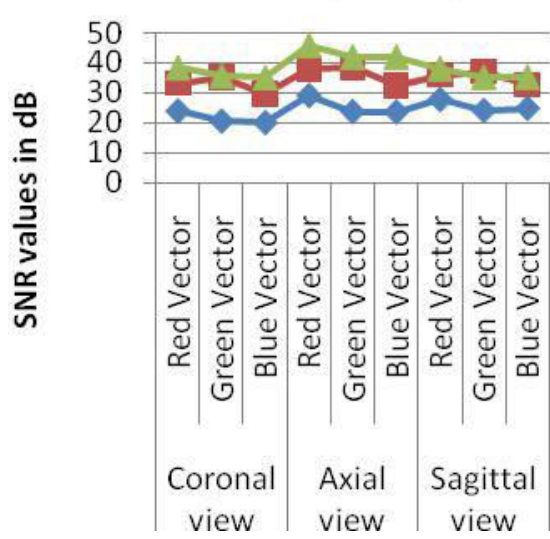

Figure 6. SNR plot showing the performance of filters for PET-CT image

\section{CONCLUSION}

In this paper, the comparative analysis of $\mathrm{PET} / \mathrm{CT}_{[13]}$ images for the preprocessing stage is done. The filtering using different filters are performed and their metrics are compared shown in Table 1. From the metrics, wiener filtering method gives the highest PSNR and SNR values and least MSE and RMSE values. The SNR plot for different filters is shown in figure 6. The contrast enhancement for hue, saturation and intensity component images are compared and concluded that equalized saturation component of HSI has larger values for CNR and lowest values for MAE and AMBE metrics shown in Table II. From the results, it is concluded that the saturation and hue component of HSIcan give the better resultsfor further processing like segmentation or classification of tumor in $\mathrm{PET} / \mathrm{CT}$ images.

\section{REFERENCES}

1] J. B. Antoine Maintz and Max A. Viergever. "A survey of medical image registration". Image Sciences Institute, Utrecht University Hospital, Utrecht, The Netherlands, Medical Image Analysis (1998) volume 2, number 1, pp 1-36 c Oxford University Press at Elsevier journal.

2] Brent Foster, UlasBagci n, AwaisMansoor, ZiyueXu, Daniel J. Mollura. "A review on segmentation of positron emission tomography images". Computers in Biology and Medicine 50 (2014) 76-96 at Elsevier journal.

[3] S. Basu, T. Kwee, S. Surti, E. Akin, D. Yoo, A. Alavi, "Fundamentals of PET and PET/CT imaging", Ann. NY Acad. Sci. 1228 (1) (2011) 1-18.

4] David Jakobsson and Fredrik Olofsson Supervisors: Anders Ericsson and Johan Karlsson. "Decision Support System for Lung Cancer using PET/CT Images". Lung Institute of Technology Andreas Jarund, WeAidU in Europe AB7th October 2004.

[5] Kim SK, Allen-Auerbach M, Goldin J, "Accuracy of PET/CT in characterization of solitary pulmonary lesions". J Nucl Med. 2007;48:214-20.

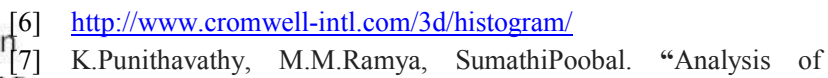
Statistical Texture Features for Automatic Lung Cancer Detection in PET/CT Images" In International Conference on Robotics, Automation, Control and Embedded Systems - RACE 2015

8] P.Nivetha1, Mr.R.Manickavasagam, "Lung Cancer Detection at Early Stage Using PET/CT Imaging Technique" International Journal of Innovative Research in Computer and Communication Engineering, Vol. 2, Issue 3, March 2014

Filterin[9] Mr. Sandeep A. Dwivedi, Mr. R. P. Borse1, Mr. Anil M. Yametkar, "Lung Cancer detection and Classification by using Machine Learning \& Multinomial Bayesian" IOSR Journal of Electronics and Communication Engineering (IOSR-JECE) Volume 9, Issue 1, Ver. III (Jan. 2014), PP 69-75

[10] NourhanZayed and Heba A. Elnemr "Statistical Analysis of Haralick Texture Features to Discriminate Lung Abnormalities".International Journal of Biomedical Imaging, Volume 2015 (2015)

[11] K.N. Plataniotis and A.N. Venetsanopoulos "Color Image Processing and ApplicationsEngineering” Monograph (English), February 18, 2000 Springer

[12] H.D. Cheng*, X.H. Jiang, Y. Sun, Jingli Wang, "Color image segmentation: advances and prospects" Published by Elsevier Science Ltd. September 2000 Pattern Recognition Society.

13] Gomes, J., Velho, L."Image Processing for Computer Graphics". Springer Verlag, New York, N.Y., 1997

14] Images Neethu M. Sasi, V. K. Jayasree Govt. Model Engineering College, Cochin University of Science and Technology,"Contrast Limited Adaptive Histogram Equalization for Qualitative Enhancement of Myocardial Perfusion" Scientific research at may 2013. 